Revue d'histoire de l'Amérique française

RAS REVUE D.HISTOIRE DE L'AMÉRIQUE FRANÇAISE

\title{
La vie aventureuse de Daniel Greysolon, sieur Dulhut
}

\section{Antoine d'Eschambault}

Volume 5, numéro 3, décembre 1951

URI : https://id.erudit.org/iderudit/801714ar

DOI : https://doi.org/10.7202/801714ar

Aller au sommaire du numéro

Éditeur(s)

Institut d'histoire de l'Amérique française

ISSN

0035-2357 (imprimé)

1492-1383 (numérique)

Découvrir la revue

Citer cet article

d'Eschambault, A. (1951). La vie aventureuse de Daniel Greysolon, sieur Dulhut. Revue d'histoire de l'Amérique française, 5(3), 320-339.

https://doi.org/10.7202/801714ar d'utilisation que vous pouvez consulter en ligne.

https://apropos.erudit.org/fr/usagers/politique-dutilisation/ 


\section{LA VIE AVENTUREUSE DE DANIEL GREYSOLON, SIEUR DULHUT}

En avril 1672, Louis XIV déclarait la guerre à la Hollande. Les années précédentes avaient été remplies de préparatifs militaires et de démarches diplomatiques en vue de l'événement. Jamais bien considérables, les secours à la Nouvelle-France avaient été complètement interrompus ${ }^{1}$. Or, ce fut précisément à cette époque que l'empire français d'Amérique, laissé à lui-même, prit un nouvel essor et atteignit des proportions gigantesques.

Le 4 juin 1671 , grâce à Nicolas Perrot et à son emprise sur les tribus des Grands Lacs, M. de Saint-Lusson, délégué de Talon, prenait possession de "tout le pays jusqu'à la Mer du Sud". Cette cérémonie se passait au Sault-Sainte-Marie en présence des chefs de quatorze nations réunies, des missionnaires, soldats, traitants et voyageurs, au cours d'une cérémonie marquée de pompe et de grandeur. Quelques mois plus tard, Jolliet et Marquette, par la découverte du Mississipi, aidaient à fixer les limites occidentales de l'empire français d'Amérique. Il restait à consolider cet immense territoire. En 1679 Dulhut faisait alliance avec les Sioux et prenait possession de leur pays; en 1682, c'était au tour de La Salle de planter les armes de France à l'embouchure du grand fleuve, et en 1689 Nicolas Perrot revenait sur la scène et annexait à la Couronne de France, le haut du Mississipi. Ainsi, dans l'espace d'une dizaine d'années, toute cette région, jusque là inconnue, passait sous le gouvernement de la Nouvelle-France par la seule influence d'une poignée de Français et de Canadiens qui se trouvaient dans cette partie du pays.

A ces explorateurs, traitants ou aventuriers: Perrot, les Tonti, La Durantaye, Louvigny, La Salle, Péré, revient, en effet, le rare mérite d'avoir conquis le respect et souvent l'affection des Indiens

1. Abbé Lionel Groulx, La Naissance d'une Race (Montréal, 1938), 17. 
des Lacs: tribus disparates toujours prêtes à la guerre, jalouses de leur autonomie, travaillées en plus par l'ennemi. Peu s'en est fallu que cette région ne passât alors aux Anglais et que la NouvelleFrance ne se vít réduite à l'étroite bande du Saint-Laurent. A travers ces années de misère et de gloire, l'hégémonie française fut maintenue au pays des Lacs par quelques hommes intrépides, courageux hérauts, parmi lesquels nous voudrions faire sa place à Daniel Greysolon, sieur Dulhut.

Il naquit à Saint-Germain-en-Laye, en 1639 ou 1640. Sa famille serait originaire de Forez, ancien pays de France baigné par la Loire ${ }^{2}$. Il semble avoir vécu à Lyon, dans sa jeunesse, d'où le nom de "Lyonnais" quı lui est parfois attribué ${ }^{3}$. Thwaites affirme, se fondant sur Tanguay, qu'il était de religion "réformée"; rien ne semble justifier cette assertion. Son testament fait un an avant sa mort, soit en 1709 , est celui d'un catholique pratiquant ${ }^{4}$. Il est donné comme enseigne dans le régiment lyonnais, en 1657, et comme gendarme de la Maison du Roi, en 1664. Cette dernière qualité prouverait sa haute naissance, puisqu'il fallait témoigner d'au moins deux cents ans de noblesse pour être de la gendarmerie de la Maison du Rois.

Dulhut affirme avoir fait deux voyages au Canada avant de s'y établir à demeure. Au retour de son second voyage il fit la campagne de Franche-Comté et prit part au combat de Senef, comme écuyer du Marquis de Lassay. Cette bataille de Senef, qui dura la journée du 11 aout 1674, fut excessivement rude. Condé y rencontrait Guillaume d'Orange; le prince et le Marquis de Lassay eurent respectivement trois et deux chevaux tués sous eux ${ }^{6}$. Après

2. Sur les origines de Dulhut, voir: R.P. L. Le Jeune, Dictionnaire géneral... du Canada (2 vol., Ottawa, 1931), au mot Dulhut; Pierre Margry, Découvertes et érablissements des Français dans l'ouest et dans le sud de l'Amerique septentrionale (1614-1754) Mémoires et documents originaux (6 vol., Paris, 1879-1888), VI: II, Introduction.

3. La Hontan parle de lui comme "gentilhomme lyonnais". Parkman le fait naitre à Lyon.

4. Reuben Gold Thwaites, The Jesuit Relations and Allied Documents (73 vol., Cleveland, 1881-1901), 62: 274.

5. Archives Publiques du Canada, C 11A (2), vol., 120: 29. Voir aussi, William McLennan, "The Death of Duluth", Mémoires et comptes rendus de la Société Royale du Canada (Toronto, 1903), 39, section II.

6. Pierre Margry, Découvertes et etablissements des Français... VI: 21; William McLennan, ibid., 12. 
Senef, Dulhut nous apprend qu'il "repartit pour retourner à Québec."7 Il serait donc revenu en Nouvelle-France, soit à l'automne de. 1674 ou au printemps de 1675 . Il avait au Canada plusieurs amis ou parents: un oncle bien connu, puissant marchand de Montréal, Jacques Patron; un beau-frère, Louis Tayon, sieur de Lussigny, officier de la garde de Frontenac et il était cousin des célèbres frères Alphonse et Henri de Tonti ${ }^{8}$. Il remenait avec lui son frère plus jeune, Claude Greysolon, sieur de La Tourette. A Montréal, il acheta de Pierre Pigeon une propriété sise sur la rue Saint-Sulpice, près de la rue Notre-Dame d'aujourd'hui'.

Dulhut affirme avoir eu, dès son premier voyage en NouvelleFrance, le dessein de se rendre chez les Sioux. Projet qu'il entreprit d'exécuter après son retour en 1674 ou 1675, "pour tascher de faire la descouverte des Nadouesioux et Asseniboualaks qui nous estaient inconnus, et leur faire faire la paix avec toutes les nations du tour du lac Supérieur, qui vivent sous la domination de nostre invincible monarque"10. Dans cette même lettre au ministre, Duluth se défend d'avoir fait la traite des fourrures au cours de ce premier voyage. Il est cependant moins catégorique dans sa lettre à Frontenac, datée du 5 avril 1679, où ll est question de son "hivernement, cabané dans le bois, $\mathbf{a}$ trois quarts de lieue de Sainte-Marie du Sault."11 Parlant des tribus du nord (Assiniboines), il y note de quel intérêt serait un commerce des habitants de la Nouvelle-France avec ces Cris et Assiniboines. "Si quelques François n'estoient allez au Nord, à la fin de l'année dernière, affirme Dulhut, tous les sauvages dudit lieu auroient porté les pelleteries à la baye d'Hudson."'12 Il est probable que le voyageur n'était pas étranger à ce commerce du nord, comme nous aurons l'occasion de le dire plus loin.

7. Pierre Margry, ibid., VI: 21.

8. Gérard Malchelosse, "Les coureurs de bois au XVIIe siècle", le Cahier des Dix (Montréal, 1941), 131. Aussi William McLennan, "The Death of Duluth", Mémoires de la Société Royale du Canada (Toronto, 1903), 15. Parkman affirme qu'il était beau-frère de Louvigny. Il semble confondre avec le Sieur de Lussigny. 39.

9. Victor Morin, "Les fastes de Montréal", le Cahier des Dix (Montréal, 1944),

10. Dulhut à Seignelay, Pierre Margry, Découvertes et établissements des français... VI: 21 .

11. Ibid., VI: 34 .

12. Ibid., VI: 30 . 
L'Intendant Duchesneau a accusé Dulhut d'avoir fait clandestinement la traite et l'a qualifié de "chef de party" parmi les coureurs de bois ${ }^{13}$. Plus tard l'intendant n'hésitera pas à soutenir que "le gouverneur, les Sieurs Perrot, Boiseau, Dulhut et Patron envoient des fourrures aux Anglais." ${ }_{14}$ La Salle, encore plus acerbe, affirme, de son côté, qu'après avoir "coulé son navire, le "Griffon", et s'être emparé des marchandises, le pilote s'est enfui vers le Haut-Mississipi, dans l'espoir de rejoindre Dulhut". ${ }^{15}$ Dans un long réquisitoire a Seignelay, La Salle charge Dulhut de maintes accusations: il aurait ouvertement bravé les ordonnances royales, en pratiquant la traite illicitement (chose que La Salle pratiquait lui-même dans la même région); il se serait fait passer pour l'agent du gouverneur au cours de négociations déjà entamées entre Sioux et Sauteux; il aurait revendiqué l'honneur de la découverte du pays des Sioux, alors qu'en réalité les hommes de La Salle, Hennepin et Accault, en auraient été les premiers explorateurs ${ }^{16}$.

Même dans l'hypothèse d'un voyage chez les Sioux qui n'aurait été "rien autre qu'une affaire de traite organisée""17, - ce qui reste à établir - l'expédition de Dulhut demeure-t-elle sans importance pour le prestige du nom français?

Les Sioux du Mississipi étaient à cette époque encore très peu connus des Français ${ }^{18}$. Radisson les avait visités en 1659 et euxmêmes lui avaient rendu sa politesse en $1660^{19}$. Quelques années plus tard, en 1665, ils avaient rendu visite au Père Allouez, au même endroit, à Chagouamigon, à la mission du Saint-Esprit ${ }^{20}$. Mais la

13. Ibid., VI: 24.

14. Archives canadiennes, Supplement (1899), lettre du 13 novembre 1681, 39.

15. Francis Parkman, La Salle and the Discovery of the Great West (Boston, 1885), 182-183.

16. Pierre Margry, Découvertes et établissements des Français... II : 246-262.

17. Gérard Malchelosse, "Les Coureurs de bois au XVIIe siècle", le Cahier des Dix (Montréal, 1941), 131.

18. Les Français connaissaient mieux les Winnebagos ou "Puants", tribu siouse dont La Potherie a laissé une peinture peu flatteuse. Les "Puants" habitaient alors le secteur de la Baie des Puants (Green Bay).

19. Grace Lee Nute, Caesars of the Wilderness (New York, 1943), 64.

20. P. Camille de Rochemonteix, Les Jesuites et la Nouvelle-France au XVIIe siècle (3 vol., 1895-1896), 2: 358 . 
légèreté ou peut-être la ruse des Hurons et Outaouais, établis dans le secteur de Chagouamigon, avaient compromis ces beaux débuts. En 1671 les Sioux avaient chassé de l'endroit ces voisins peu sûrs ${ }^{21}$. Les négociations furent cependant reprises. En 1674 dix délégués Sioux vinrent au Sault-Sainte-Marie dans le but de faire la paix avec les Outaouais. Cette fois, ce fut au tour des Cris de s'opposer a la paix; résolus à mettre à mort les délégués, ils finirent par pénétrer dans l'abri où les Jésuites avaient caché les malheureux. Un massacre général s'ensuivit ${ }^{22}$. Les Sioux se montrèrent si irrités de ces machinations que, durant les années qui suivirent, la prudence interdit de s'aventurer dans la partie nord-ouest du lac Supérieur ${ }^{23}$. Telle est la situation décrite par Dulhut, dans sa lettre à Frontenac, quand il dit: Ils (les Saulteurs et autres sauvages) [ pourront ] lorsqu'ils seront en paix, chasser autour du fond du Lac, ce qu'ils n'osent faire présentement,... si bien que ce lieu est une pépinière de castors, veu que depuis plus de dix ans personne n'y a chasse." 24

A la lumière de ces faits on comprend mieux l'importance des expéditions de Dulhut. Les Sioux étaient les gardiens naturels de la vallée du Mississipi et des régions limitrophes, très riches en fourrure, régions que Talon venait de choisir comme limite occidentale de l'empire d'Amérique ${ }^{25}$. On croyait, en plus, surtout depuis Radisson, - et Dulhut partageait cette illusion - que la découverte de la Mer de l'Ouest devait se faire par le pays des Sioux ${ }^{26}$.

Le 1er septembre 1678, Dulhut quitte Montréal avec sept compagnons: son frère, La Tourette, les deux frères Pépin, les sieurs Le Maistre (ou Lemaître), Paul Lavigne, Bellegarde et La Rue ${ }^{27}$, ainsi

21. Ibid., 3: 7 .

22. Thwaites, The Jesuit Relations and Allied Documents, 58: 256-262.

23. Louise Phelps Kellogg, French Regime in Wisconsin and the Northwest (Madison, Wisconsin, 1925), 209.

24. Pierre Margry, Découvertes et établissements des Français... VI: 30.

25. P. Camille de Rochemonteix, Les Jésuites et la Nouvelle-France au XVIIe siècle (3 vol., 1895-1896), 3: 10.

26. Grace Lee Nute, Caesars of the Wilderness (New York, 1943), 318.

27. Louise Phelps Kellogg, French Regime in Wisconsin and the Northuest (Madison, 1925), $209-$ Gérard Malchelosse, "Les coureurs de bois au XVIIe siècle", Le Cahier des Dix (Montréal, 1941), 132. 
que trois sauvages prisonniers que des Indiens amis lui ont donnés comme esclaves ${ }^{28}$. Dulhut et ses hommes se rendent près du SaultSainte-Marie où ils décident d'hiverner. Il semble que l'hiver se passe à rencontrer les Indiens et vraisemblablement a faire la traite. Selon plusieurs auteurs, Dulhut aurait établi, durant cet hiver de 1678-1679, un poste à l'embouchure de la rivière Kamınistiquia, sur l'emplacement actuel de Fort William, tandis que son frère ouvrait un comptoir a l'embouchure du lac Nipigon ${ }^{29}$. Comme Dulhut n'affirme rien à ce sujet, nous en restons à des conjectures. Mais il fait mention d'un voyage de "quelques Français partis faire la traite dans le Nord" et il justifie leur action; d'autre part, son frère et quelques autres des siens ne l'ont pas accompagné à son voyage chez les Sioux, au printemps de 1679; il semble donc probable qu'en effet La Tourette établit un poste au lac Nipigon durant cet hiver. Quant au poste de Kaministiquia, nul document n'en atteste l'existence à cette date.

Le 15 décembre 1678, Dulhut rencontre Oumamime, chef Sauteux, qui lui apprend que les Sauteux et les Sioux ont déjà entamé des pourparlers, mais que sans l'appui des Français aucune paix ne pourrait être durable. Il est convenu que Dulhut accompagnera les Sauteux chez les Sioux et qu'on enverra des Français vers les Assiniboines et autres tribus du Nord, pour les avertir de la paix en train de se conclure. Au printemps de 1679 Dulhut part donc avec quatre de ses premiers compagnons: Le Maistre, Bellegarde, les frères Pépin et un nommé Masson, interprète, ainsi que les délégués Sauteux pour aller rencontrer les envoyés Sioux au rendez-vous convenu, au fond du lac Supérieur ${ }^{30}$. La ville actuelle du Dulhut serait l'endroit probable où se rencontrèrent les représentants des deux tribus, alors les plus puissantes de l'Ouest ${ }^{31}$.

Les pourparlers furent couronnés de succès. Dulhut décida d'accompagner les Sioux dans leur pays. Et c'est là que, le 2 juillet

28. Pierre Margry, Découvertes et établissements des Français... VI: 21.

29. A.J. McCowber, "Some Early History of Thunder Bay and District", Publications de la Thunder Bay Society (1923-1924), 13. - Louise Phelps Kellogg, French Regime in Wisconsin and the Northwest (Madison, 1925), 226.

30. Pierre Margry, Découvertes et établissements des Français... VI: 29.

31. Grace Lee Nute, Lake Superior (New York, 1944), 29. 
1679, il prit possession de cette région, au nom du roi de France. La carte de Hennepin, de 1683 , désigne le chêne sur lequel Dulhut plaça l'écusson royal ${ }^{32}$. L'endroit visité par Dulhut est décrit comme "le grand village des Nadouesioux, appelez Izatys, où jamais François n'avoit esté, non plus qu'aux Songaskikons et Houetbatons, distans de ces premiers de six vingtz lieues, où j'ay aussy arborer les armes de Sa Majesté, dans la mesme année 1679"'33. Le gros bourg sioux était situé sur les bords d'un lac que Dulhut nomme "lac Buade" et qui est aujourd'hui connu sous le nom de Mille Lacs, au sudouest du lac Supérieur. Les tribus rencontrées par Dulhut et désignées sur la carte de Hennepin, à l'est du lac Mille Lacs, pourraient être celle des Dakota-Sissitons (habitans du riz sauvage) et celle des Dakota-Wahpetons (habitants des forêts) ${ }^{34}$. Dulhut qui a fait le trajet du fond du lac au pays des Sioux à pied, visite les autres villages de la même manière et paraît y employer une bonne partie de l'été de 1679. Le 15 septembre de la même année, c'est au tour des Assiniboines et autres tribus du nord de se rencontrer avec les Sioux, encore au fond du lac Supérieur, et de faire la paix. Dulhut s'efforce, durant les mois qui suivent, de cimenter ces alliances par trop éphémères. Encore ici il continue, selon toute probabilité, de faire la traite et il est fort possible qu'il ait ouvert alors un comptoir à l'embouchure de la rivière Kaministiquia. En outre il a obtenu des Sioux, Assiniboines et tribus du nord, la promesse qu'une délégation aille visiter Frontenac au printemps suivant. Une épidémie de petite vérole empêche la réalisation de cette promesse ${ }^{35}$.

Dulhut, semble-t-il, laisse trois de ses hommes chez les Sioux, à sa visite de 1679 . Comme tous les Français du temps, il se croit à proximité de la Mer de l'Ouest et vraisemblablement, il souhaite connaître, par leur entremise, la meilleure route à suivre pour s'y rendre. Les engagés de Dulhut rendent visite à une tribu siouse qui a été guerroyer à vingt jours de marche, et après avoir rencontré une

32. Louise Phelps Kellogg, French Regime in Wisconsin and the Northwest (Madison, 1925), face à page 210 .

33. Pierre Margry, Découvertes et établissements des Français... VI: 22.

34. Louise Phelps Kellogg, French Regime in Wisconsin (1925), 211. - Gontran Laviolette, O.M.I. The Sioux Indians in Canada (Regina, Sask., 1944), 12.

35. Louise Phelps Kellogg, ibid., 211. 
autre tribu, a pu atteindre un grand lac à l'ouest, d'où ils ont rapporté du sel. On a supposé que la tribu rencontrée par les Sioux était celle que Hennepin désigne sous le nom de "Tintons" sur sa carte. Ce pourrait être les Tetons et la mer salée pourrait être le célèbre Lac Salé de la légende et de l'histoire. Les hommes de Dulhut auraient rencontré la tribu siouse au lac Big Stone et auraient été les premiers blancs à fouler le sol du Dakota, après avoir traversé le Minnesota ${ }^{36}$.

Une fois la paix établie, Dulhut résolut de pénétrer plus à l'ouest, en utilisant les renseignements recueillis par ses hommes: "Mon dessein fust de pousser jusques à la mer du costé de l'ouestnord-ouest, qui est celle que l'on croit estre la mer Vermeille ${ }^{37}$, d'où les Sauvages, qui estoient allez en guerre de ce costé-là, donnèrent du sel à trois François, que j'avois envoyez à la descouverte..." ${ }_{38}$

Dulhut s'aventura sur une route jusque alors inconnue des Français. La description qu'il en a faite, s'accorde avec ce qu'en dit La Salle. Après avoir remonté la rıvière Brûlé qui se jette dans le lac Supérieur, "à huit lieues du fond du lac", il s'engage bientôt sur les eaux de la rivière Sainte-Croix qui, celle-ci, dégorge dans le Mississipi. Dulhut mène avec lui, dans deux canots, quatre Français et un sauvage interprète ${ }^{39}$.

A l'embouchure de la rivière Sainte-Croix, l'explorateur apprend une nouvelle qui lui fait changer ses plans: "J'appris par huit cabanes de Nadouesioux, que j'y rencontray, que le Révérend Père Louis Henpin, Recollect, de présent au couvent de Saint-Germain, et deux autres François, avoient esté volez et menez en esclaves pendant plus de 300 lieues par les Nadouesioux mesmes." ${ }_{40}$ Il est pro-

\section{Ibid, 211.}

37. La "Mer vermeille" désignait en réalité le golfe de Californie. Les premiers explorateurs confondirent longtemps les deux: le golfe et la Mer de l'Ouest proprement dite. Le mot "vermeille" était une corruption française de l'expression espagnole "mar Bernejo" ou "mer rouge", à cause de sa ressemblance en couleur et en forme, avec la Mer Rouge de l'Ancien Testament. Les Anglais disaient de leur côté, "Vermilion Sea". Voir Thwaites, The Jesuit Relations and Allied Documents, 59: 307.

38. Pierre Margry, Découvertes et établissements des Français... VI: 24.

39. Ibid., 2: 249-254. - Louise Phelps Kellogg, French Regime in Wisconsin and the Northwest (Madison, 1925), 336.

40. Pierre Margry, Découvertes et établissements des Français... VI: 23. 
bable toutefois qu'à ce moment précis, Dulhut ait ignoré que l'un des prisonniers fât un religieux. Parkman affirme qu'il croyait à la présence de traitants anglaị ou espagnols dans le Haut-Mississipi, et qu'il décida immédiatement d'aller s'enquérir ${ }^{41}$. Il partit donc avec deux des Français et un sauvage, et après avoir navigué sans trêve durant deux jours et deux nuits, il rejoignit la bande de Sioux, au nombre de 1,000 ou 1,100, dit-il, qui tenaient le Père Hennepin, Michel Accault et Antoine Auguelle prisonniers ${ }^{42}$. Hennepin avait quitté le fort Crèvecœur de La Salle en février, en route vers le Haut-Mississipi, dans un but d'exploration et de traite ${ }^{43}$. En avril, les trois Français avaient été faits prisonniers par les Sioux. Ils avaient ainsi remonté le fleuve, plus haut que les Chutes SaintAntoine, où se trouve aujourd'hui la ville de Minneapolis et s'étaient approchés des sources du Mississipi. Dulhut les rejoignit, soit en aval de l'embouchure de la rivière Sainte-Croix, peut-être aux environs de l'embouchure de la rivière Wisconsin; peut-être aussi tout à fait au nord, près des sources du Mississipi, le 25 juillet $1680^{44}$.

Dulhut revint avec les guerriers Sioux à leur village qui était probablement celui de Mille Lacs. Il reprocha à la tribu assemblée sa trahison et déplora amèrement ce qu'il appelait leur "insulte". Cependant il se sépara des Sioux en bons termes et revint avec ses nouveaux compagnons à Michilimakinac, en passant par la Baie Verte (mission Saint-François-Xavier). Le retour se fit, semble-t-il, par les rivières Wisconsin et des Renards. En ce cas, Dulhut aura été le premier blanc à explorer ces deux importants cours d'eau ${ }^{45}$.

En arrivant à Michilimakinac il apprend que l'intendant $\mathrm{Du}-$ chesneau l'accuse d'être le chef des coureurs de bois rebelles. En réalité il n'a jamais eu plus que huit hommes avec lui. Dulhut décide immédiatement de se rendre à. Québec pour se disculper. Il part 276.

41. Francis Parkman, La Salle and the Discovery of the Great West (Boston, 1885),

42. Pierre Margry, Découvertes et établissements des Français... VI: 23.

43. Voir, pour les motifs de ce voyage, Grace Lee Nute, Caesars of the Wilderness (New York, 1943), 183.

44. Il nous semble plus probable, à la lumière des quelques documents existants, que Dulhut rencontra les captifs en amont du fleuve.

45. Louise Phelps Kellogg, French Regime in Wisconsin and the Northrest (Madison, 1925), 213. 
avant le dégel, le 29 mars 1681 , avec deux compagnons, traînant son canot et ses vivres sur les glaces ${ }^{46}$. A Québec l'intendant refuse de l'entendre et Frontenac, comme il l'écrit à Colbert, doit le garder chez lui afin de le protéger. Le gouverneur le traite avec beaucoup d'amitié et lui conseille de passer en France.

Nous retrouvons Dulhut à Paris où il présente au marquis de Seignelay une requête en vue d'établir un poste chez les Sioux, avec droits seigneuriau $x^{47}$. La supplique de Dulhut reste sans réponse. La mort de Colbert et le rappel de Frontenac, peut-on 'conjecturer, ne sont pas étrangers à cet insuccès. D'ailleurs une instruction du roi, en date précisément de mai 1682, vise à restreindre l'ardeur des explorateurs et traitants. "Sa Majesté, disait le rescrit, n'estime pas que ces descouvertes soient advantageuses... elle donne ordre audit sieur De la Barre de ne pas continuer à donner de pareilles permissions, mais seulement de laisser achever celle commencée par le sieur De la Salle jusques à l'embouchure de ladite Rivière de Mississipy." 48 Dulhut s'embarque pour la Nouvelle-France, en cette même année de 1682, sur le vaisseau qui amène au Canada le nouveau gouverneur, M. de La Barre et le sieur de Meulles, le nouvel intendant.

Dès son retour, Dulhut apprend que la situation dans le secteur des Grands Lacs s'est considérablement empirée. Le 10 octobre 1682, il assiste à une importante réunion tenue chez les Pères Jésuites à Québec. Le nouveau gouverneur, M. de La Barre, y est présent ainsi que l'intendant, avec Monseigneur de Laval, M. de Varennes, le père du futur découvreur de l'Ouest canadien et $M$. de La Durantaye. Dulhut a sans doute été invité à cause de sa connaissance des pays d'En-Haut. Les minutes de cette assemblée nous ont été conservées. Les Iroquois, y lit-on, essaient "de détruire toutes les nations nos alliés les unes après les autres... pour après qu'il nous auront ôté tout le commerce des pelleteries qu'ils veulent seuls faire avec les Anglais et Hollandais établis a Manatte et Orange, nous attaquer seuls et ruiner la Colonie en l'obligeant de se resserrer et quitter toutes les habitations séparées, et ainsi faire cesser la culture

46. Pierre Margry, Découvertes et établissements des Français... VI : 25.

47. Ibid., VI: 35-36.

48. Ibid., VI: 37 . 
des terres... Depuis quatre ans les Anglais n'ont rien omis pour engager les Iroquois à nous déclarer la guerre, soit par le grand nombre de présens qu'ils leur ont faits soit par le bon marché auquel ils leur ont donné les denrées et surtout les fusils, la poudre et le plomb..." ${ }_{49}$

La Nouvelle-France faisait face à un double danger qui menaçait sa vie économique et son existence même. En faisant disparaître les Hurons, les Iroquois avaient espéré se substituer à ces entremetteurs des comptoirs français et diriger la traite des fourrures vers le marché anglais. Mais ils se sont bientôt heurtés aux tribus des Grands Lacs ralliées par les missionnaires, les commandants et traitants français; ce qui a freiné, sinon refoulé leur mouvement d'expansion. En 1671 , ils ont vu M. de Courcelle naviguer sur le lac Ontario qu'ils avaient cru jusque là à l'abri des blancs, et presque en même temps, Frontenac faisait construire le fort Cataracoui en plein territoire iroquois. Un autre danger encore plus sérieux a surgi. C'est à peu près à cette époque que les traitants anglais, surtout ceux de la Virginie, commencent à envahir la vallée du Saint-Laurent. Détruire les tribus alliées des Français, et par les Iroquois, se rendre maitres de la traite, tel est leur plan. En 1680 les Iroquois attaquent les Illinois, qui occupent la région-est du Mississipi, le long de la rivière des Illinois. Immédiatement Frontenac exige des réparations et permet aux tribus alliées de se défendre contre les Iroquois. Dans le but de protéger les Illinois, La Salle et Tonti construisent le célèbre fort Saint-Louis, sur "le Rocher". Ils projettent aussi d'établir une première colonie sur les rives de la rivière des Illinois ${ }^{50}$. La fière attitude de Frontenac rallie d'abord les tribus algonquines et les Hurons. Mais le rappel du gouverneur occasionne un fléchissement. De La Barre, jaloux de La Salle, fait saisir, dès son arrivée, le fort Frontenac et envoie le chevalier de Baugy occuper le fort Saint-Louis.

C'est en ces conjonctures que Dulhut revient dans l'Ouest canadien, en 1683. Cette fois il s'est muni d'un permis de traite. Est-il vrai qu'il a été du parti du chevalier de Baugy, dans la saisie du fort Saint-Louis et qu'au retour on l'a chargé de fortifier Michilimaki-

49. Thwaites, The Jesuit Relations and Allied Documents, 62: 156-158.

50. Pierre Margry, Découvertes et établissements des français... II: 186. Voir aussi Francis Parkman, La Salle and the Discovery of the Great West, 313-314. La Salle rêvait d'établir une seconde colonie à l'embouchure du Mississipi. Voir aussi, S.E. Mgr. Schlarman, From Quebec to New Orleans (Belleville, Illinois, 1929), 92s. 
nac $?^{51}$ Le Père Enjalran, qui écrit de Michilimakinac, le 26 août 1683, permet de présumer que Dulhut n'a pu prendre part à l'expédition. Il n'a pu arriver à temps et le chevalier de Baugy a d'ailleurs suivi une autre route. Selon le même informateur, Dulhut se dirigeait déjà vers le pays des Sioux avec 30 hommes et le Père Pierson, jésuite, qui servait de chapelain. Les Français devaient laisser leurs marchandises à la mission Saint-François-Xavier, à la Baie des Puants, et marcher immédiatement vers les Poutéatamis pour leur reprocher leur attitude envers les Français. Dulhut devait en plus essayer d'établir une alliance entre les Ouatagamis et les Sauteux ${ }^{52}$. Il semble cependant que Dulhut a, cette fois encore, pour principale préoccupation, de gagner les Sioux à l'allégeance française. Rêve-t-il encore

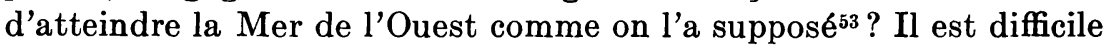
de le dire. Dulhut prend la route des rivières des Renards et Wisconsin, remonte le Mississipi jusqu'à l'embouchure de la rivière SainteCroix, et retrouve ses alliés Sioux qui le reçoivent à bras ouverts, bien qu'ils aient, dès 1680, brisé leur première alliance. Dulhut semble avoir hiverné au portage, entre les rivières Sainte-Croix et Brûlé, près de Solon Springs d'aujourd'hui où il établit un poste de traite ${ }^{54}$.

Dulhut se trouve alors à proximité du lac Supérieur; il est possible qu'il y ait construit le poste de Kaministiquia ${ }^{55}$. Et ici se place un incident qui peint le courage et le sang-froid du héros. Deux Français, Jacques Le Maire et Colin Berthot, ont été tués par des Sauteux à la baie Keweenaw. Dulhut apprend probablement par le Père Albanel, que l'un des assassins est arrivé au Sault-SainteMarie, mais que les Français de l'endroit, au nombre de douze seule-

51. R.P L. Le Jeune, Dictionnaire gêneral du Canada (2 vol , Ottawa, 1931).

52. Lettre du Père Enjalran, s.j. (Michillimakinac), Pierre Margry, Découvertes et etablissements des français... V: 3-7.

53. Grace Lee Nute, Lake Superior (New York, 1944), 29.

54. Ibid., 29; Louise Phelps Kellogg, French Regime in Wisconsin and the Northwest (Madison, 1925), 225; La carte de Franquelin, de 1688, montre l'établissement de Dulhut, nommé "Fort Ste-Croix".

55. "Il y a encore plusieurs postes occupés par les Français dans le lac Supérieur L'un des plus anciens est celui de Kaministiquia, situé du côté nord. Ce poste a été construit, en 1683, par le défunt Daniel de Greysolon, Sieur du Luth. Claude de Greysolon Sieur de la Tourette, y a commandé". Mémoire du Sieur Boucault, 1754, Rapport de l'Archiviste de la Province de Québec (1920-1921): 13. 
ment, n'ont pas osé l'arrêter. Le voyageur part immédiatement de son poste, soit de Kaministiquia ou de la rivière Sainte-Croix; il prend avec lui six Français, se rend en vitesse au Sault-Sainte-Marie. Sans hésitation il fait arrêter et garder le sauvage, nommé "La Folavoine". Puis, aidé de Jean Péré, il fait aussi arrêter les enfants du chef Sauteux, Achiganaga. Péré mène avec lui dix-huit voyageurs qui ont hiverné à Keweenaw. On instruit le procès; on interroge séparément les prisonniers, puis collectivement. Les hommes de la tribu eux-mêmes sont appelés à décider du sort des deux Indiens reconnus pour les plus coupables: La Folavoine et l'aîné des fils d'Achiganaga. "A la tête de 42 Français, dit Dulhut, et en présence de 400 sauvages, je leur fis casser la tête." ${ }_{56}$

En même temps que ces événements se passent dans la région des Grands Lacs et que la lutte entre Français et Anglais se fait plus vive, la Baie d'Hudson est le théâtre d'un semblable conflit. Précisément, en janvier 1683, l'ambassadeur français proteste auprès de la cour d'Angleterre, contre les établissements anglais à la Baie d'Hudson $^{57}$. En 1682, Jean-Baptiste Chouart, second fils du célèbre des Groseillers, se trouve sur la rivière Hayes, au service des Français. Radisson, son oncle, vient de passer de nouveau aux Anglais, sans que Chouart le sache. En 1684 les vaisseaux anglais ramènent Chouart prisonnier en Angleterre. Chouart y passe l'hiver de 168485, mais il a formé le dessein de retourner à la Baie d'Hudson à la première occasion, et de regagner le Canada en passant par le poste de Dulhut, sur le lac Nipigon. Il sait probablement par le sieur de Comporté, alors en France, que Dulhut a reçu instruction de construire un fort dans cette région du nord du Lac Supérieur ${ }^{58}$. Poste apparemment établi au printemps ou à l'été de 1684. Dans sa lettre au gouverneur, en date du 10 septembre 1684, Duluut le désigne comme "le fort que j'ay fait faire à la Rivière à la Manne, dans le fond du Lac Alemipigon" 59 . Ce poste est habituellement décrit comme étant à l'embouchure de la rivière Ombabika. La Tourette, à ce qu'il semble, en assume le commandement. La carte de Jaillot (1685)

56. Lettre de Dulhut, AC, C 11A, 6: 231.

57. Grace Lee Nute, Caesars of the Wilderness (New York, 1943), 198.

58. Ibid., 233.

59. Pierre Margry, Découvertes et établissements des Français... VI: 51. 
l'indique comme suit: "Poste du Sieur Duluth, pour empêcher les Assiniboels et autres Sauvages de descendre a la Baie d'Hudson". La carte de Franquelin, 1695, le désigne sous le nom de "Fort la Tourette".

Dans le cours de la même année Dulhut envoie Péré, en qui il a confiance et dont il admire le courage, à la Baie d'Hudson, par la rivière Albany ${ }^{60}$. Lorsque Péré atteint la Baie d'Hudson, au lieu de trouver Chouart, il se heurte aux Anglais qui l'amènent prisonnier en Angleterre. Péré, avec ses compagnons La Croix et Desmoulins, sont probablement les premiers blancs qui aient suivi cette route intérieure vers la Baie du Nord. Quant à Chouart, nous savons qu'il revint à la Baie d'Hudson et en 1685 lui aussi fit un voyage à l'intétieur. Peut-être voulait-il encore là se rendre au poste du lac Nipigon? Les historiens de langue anglaise font grand état de Kelsey qui, en 1690, fit son voyage le long de la rivière Nelson. Cinq ans plus tôt, en 1685, Jean-Baptiste Chouart avait pénétré à l'intérieur par une route semblable. Il est malheureux que nous n'ayons pas de données plus précises sur ce voyage de Chouart ${ }^{61}$.

Durant le même été de 1684, Dulhut reçoit ordre de se rendre à Michilimakinac et de se joindre à M. de La Durantaye pour prendre part à l'expédition du gouverneur de La Barre contre les Iroquois. La rencontre est censée se faire au Niagara. On sait le lamentable résultat de cette expédition. Dans une série de réunions et de pourparlers, où ils deviennent de plus en plus insolents, les Iroquois forcent de La Barre à leur abandonner les Illinois. De La Durantaye, Dulhut et Perrot ont eu beaucoup de difficulté à persuader les sauvages alliés de se rendre au Niagara, ce qui ne les a pas empêchés de grouper près de 500 guerriers. En route un messager vient leur dire, de la part de La Barre, la conclusion de la paix ${ }^{62}$. A la suite de cette catastrophe La Barre est rappelé. "Plus dangereux pour le Canada,

60. La rivière Albany a d'abord porté le nom de Rivière Péré (ou Parray). Ainsi, la carte de Franquelin, de 1695.

61. Grace Lee Nute, Caesars of the Wilderness (New York, 1943), 2378. Pour ce qui est de Kelsey, une inscription montée sur une stèle, au Pas, Manitoba, le désigne comme "le découvreur des Prairies"... on croit rêver vraiment! Le poste du lac Nipigon fut en opération pendant la plus grande partie du régime français. Voir, par exemple, le rapport de Daigremont, en 1708, AC, C 11A, 29:87.

62. Prancis Parkman, Count Frontenac and New France under Louis XIV (Baston, 1885), 118. 
que les Iroquois eux-mêmes", a-t-on pu dire de ce gouverneur. Le Marquis de Denonville, son successeur, arrive à Québec en juin 1685. La situation dans le secteur des Grands Lacs est devenue très critique. Le prestige des Français est à son plus bas. Les tribus alliées sont consternées. Et les Iroquois font de nouvelles instances auprès des Hurons.

A l'automne de 1685 un transfuge français, Marion dit La Fontaine, offre aux Anglais d'Albany de les conduire au pays de la fourrure. Le capitaine Johannes Roseboom, décidé à profiter de l'occasion, équipe onze canots. A l'automne, tandis que les commandants français étaient dispersés dans la région, Roseboom et ses compagnons atteignent Michilimakinac et remplissent leurs canots des plus belles fourrures. Après quoi, rentrés triomphalement à Albany, ils annoncent que c'en est fait du monopole français dans cette partie du pays. Grandement alarmés de la chose, les officiers français font rapport à Denonville qui proteste immédiatement auprès du gouverneur de New-York, le célèbre Dongan. Celui-ci répond par une lettre insolente. C'est alors que Dulhut est appelé à fonder un poste sur la rivière Sainte-Claire, afin d'arrêter les Anglais au passage: poste qui prend le nom de fort Saint-Joseph. Comme le passage par le portage de Toronto est sans surveillance, sept traiteurs hollandais y passent en 1686, se rendent à leur tour à Michilimakinac et $y$ font eux aussi une traite abondante. L'année suivante, dès le printemps, le Capitaine Roseboom qui a hiverné en territoire iroquois, fait de nouveau son apparition à Michilimakinac avec 20 canots et un contingent de 50 voyageurs, presque tous de langue française, tandis qu'un second groupe, sous les ordres du Major Patrick McGregory, faisait également route vers Michilimakinac ${ }^{63}$. Au moment où les traitants anglais se croient hors de danger, ils sont faits prisonniers par Perrot et de La Durantaye, en route pour le sud du lac Ontario où Denonville a convié les commandants et les Indiens alliés, en vue d'une invasion du territoire iroquois.

Denonville est parti de Montréal avec 800 réguliers, 700 miliciens et 500 sauvages chrétiens, et avec l'espoir d'une assistance des tribus alliées des Grands Lacs. Perrot vient à sa rencontre avec un

63. Louise Phelps Kellogg, French Regime in Wisconsin and the Northwest (Madison, 1925), 230s. 
groupe d'Indiens du Wisconsin; Tonti avec un contingent d'Illinois tandis que Dulhut convie à son poste de Saint-Joseph, sur la rivière Sainte-Claire, les Mascoutins, les Renards et autres guerriers des environs. Tonti rejoint Dulhut à Saint-Joseph; et sous le commandement de La Durantaye, cette armée bigarrée s'en va opérer sa jonction avec celle de Denonville ${ }^{64}$. Les guerriers de l'Ouest sont au nombre de 400. Jamais agglomération humaine n'a été plus difficile à diriger et à maîtriser. Il a fallu toute l'emprise d'hommes rompus à cette tâche pour en venir à bout. La rencontre avec l'armée principale a lieu à la Baie Irondequoit, sur la rive sud du lac Ontario.

La campagne de Denonville ne dure que dix jours. Elle n'affecte en réalité qu'un seul des cinq cantons, celui des Tsonnontouans. Mais elle a pour effet d'exaspérer les autres Iroquois qui, dès la fin d'août, à l'instigation des Anglais, font irruption dans la colonie. Cette même campagne de 1687 a valu un regain de prestige aux Français. Elle a contribué cependant à rapprocher les Iroquois des Anglais, alors que ces derniers ambitionnent toujours, quoique sans succès, de passer pour protecteurs des cantons. Denonville croit bon d'envoyer le Père Vaillant de Gueslis à New York, y conférer avec le gouverneur Dongan, truchement officieux des Iroquois. Ces derniers, toujours avec l'appui des Anglais, renouvellent leurs efforts auprès des Hurons qui inclinent de plus en plus vers l'alliance sollicitée. Encore cette fois, toute l'influence des commandants et traitants français n'est pas de trop pour maintenir les Hurons dans les cadres de l'amitié française. Dans son rapport de 1687, Denonville souligne, à cette occasion, le grand service rendu par le poste de Dulhut à Saint-Joseph, qui a empêché les Hurons et autres alliés de se joindre aux Iroquois ${ }^{65}$.

Tandis que ces événements se passent dans l'Ouest canadien, La Tourette, frère de Dulhut, est en route pour Montréal et Québec. Le gouverneur signale sa présence en ces termes: "Le frère de du Luth, nouvellement arrivé des rivières qui sont au dessus du lac Allémipigon, m'assure avoir vu plus de 1,500 personnes arriver à

64. Ibid., 235. - R.P. L. Le Jeune, Dictionnaire général du Canada, au mot Denonville.

65. Lettre de Denonville, 8 juin 1687, AC, C 11A, 9: 31-50. 
la traite pour commercer avec lui... ce sont des peuples qui ont l'habitude d'aller aux Anglais du port de Nelson ou Rivière Bourbon, où ils disent n'avoir point été cette année par les soins du Sieur du Luth" 66 .

LaHontan qui a fait la campagne contre les Iroquois, rencontre La Tourette à une faible distance du Niagara, alors que celui-ci se dirige vers Montréal et Québec ${ }^{67}$. Peut-être est-ce à cause du voyage de La Tourette que Dulhut demande d'être alors relevé de son commandement au fort Saint-Joseph. Il y est remplacé par le célèbre baron LaHontan qui va y rejoindre Dulhut et Tonti. Il semble que la garnison de Dulhut à Saint-Joseph soit faite surtout de coureurs de bois qui se montrent heureux de laisser la place au détachement de réguliers commandés par LaHontan ${ }^{68}$. Le poste de Saint-Joseph, établi par Dulhut, à la demande de Denonville en 1686, était situé au fond du lac Huron, sur la rive ouest de la rivière Sainte-Claire, dans les environs du Port-Huron de nos jours. Dulhut en a fait une construction imposante, flanquée de bastions. Le fort domine l'entrée de la rivière qui se rétrécit à cet endroit ${ }^{69}$. Son importance stratégique a déjà été notée.

Dulhut passe l'hiver de 1687-88 dans la région des Grands Lacs. Nous retrouvons son nom de nouveau, à l'été de 1688 , alors que les Renards s'opposent à ce que l'on transporte des marchandises aux Sioux. Dulhut, à la tête de 300 hommes bien armés, leur fait entendre raison. Il aurait alors visité une dernière fois ses amis les Sioux, encore par la voje de la Rivière des Renards et du Wisconsin; et il se serait rendu ensuite chez les Assiniboines. Rêve-t-il de reprendre son voyage de découverte? Il est rappelé en Nouvelle-France au printemps de 1689 et quitte l'Ouest canadien pour ne plus y revenir.

66. AC, C 11A, 9: 106.

67. Un Outre-Mer au XVIIe siècle. Voyages au Canada du Baron La Hontan. Publié avec une introduction et des notes par M. François de Nion (Paris, 1900), 162.

68. Ibid., 171.

69. Pour la description détaillée du fort Saint-Joseph, voir le texte d'une lettre écrite à Pontchartrain, en 1686, dont l'original se trouve à la Bibliothèque du Congrès, a Washington, Wisconsin Historical Collections, vol. XVI. - Louise Phelps Kellogg, French Regime in Wisconsin and the Northwest (Madison, 1925), 127. Voir aussi Ernest Voorhis, Historic Forts and Trading Posts (Ottawa, 1930), au mot Détroit. 
En 1689, durant l'incursion des Iroquois contre Lachine et VilleMarie, Dulhut fait une sortie avec un groupe de 28 Canadiens; il rencontre un parti d'Iroquois, au lac des Deux-Montagnes, se met à leur poursuite, reçoit leur fusillade sans broncher, puis se jetant sur les canots ennemis, tue 18 hommes, en fait trois prisonniers, tandis qu'un seul peut s'enfuir ${ }^{70}$. En récompense de son exploit, Dulhut, sur recommandation de Frontenac, est nommé, en 1690, capitaine réformét ${ }^{71}$. Il s'est établi à Montréal, en compagnie de Jacques Bizard, s'y construit une maison luxueuse ${ }^{72}$. Atteint de la goutte, il y vit quelques années dans une paix relative. En 1695 Frontenac décide de le nommer commandant de l'important fort Frontenac. Dans les Relations de 1696 , le Père de Lamberville rapporte que " $\mathrm{M}^{\mathrm{r}}$. Du Luth cap. ${ }^{\text {ne }}$ dans la marine, depuis plus. ${ }^{\text {r }}$ années etant fort tourmenté delagoutte, et [ ne ] trouvant point desoulagem. ${ }^{t}$ a son mal, il fit dire une neuf. ${ }^{\text {ne }}$ en l'honneur [ de cette] bonne $X^{\text {enne }}$. [ Catherine Tekakwita ].. dont les prieres l'ont entierem. ${ }^{t}$ gueri de sa goute le 9e jour." 73 Voilà probablement la première mention dans notre histoire d'une faveur obtenue par l'intercession de la sainte iroquoise.

Quelques mois plus tard cependant, Dulhut se sent terrassé de nouveau par la maladie. Il demande à Frontenac son rappel, après environ quinze mois de séjour au Fort Frontenac. Le 25 mars 1696, il est nommé capitaine en pied. Le document officiel le qualifie de "bon officier". Ce n'est pas d'ailleurs le seul éloge qu'on lui ait décerné. M. de Champigny, intendant, dans une lettre au Ministre, parle "de ceux qui sont incapables de cupidité et qui sont inviolablement fidèles, comme MM. de La Durantaye, de Longueuil, Dulhut et de Maricourt"74. LaHontan lui-même, plutôt avare de compliments, a dit de lui: "gentilhomme qui a beaucoup de mérite"75. 275.

70. Francis Parkman, La Salle and the Discovery of the Great West (Boston, 1885),

71. AC, C 11A, 120: 29.

72. William McLennan, "The Death of Duluth", Memoires et comptes rendus de la Societé Royale du Canada, section II (Toronto, 1903), 41.

73. Thwaites, The Jesuit Relations and Allied Documents... 65: 32.

74. AC, C 11A, 16: 102.

75. Un Outre-Mer au XVIIe siècle. Voyages au Canada du Baron de La Hontan. Publiés avec une introduction et des notes par M. François de Nion (Paris, 1900), 103. 
Ses dernières années se passent à Montréal. En 1697 il est fait commandant d'une compagnie d'infanterie. Puis son nom disparait. Le 4 mars 1709, en présence de Maître Michel Le Pailleur, notaire, il signe son testament. Il y fait des dons en faveur des Récollets, des Jésuites et des Sulpiciens. Le 4 février 1710 il rédige un nouveau testament, substantiellement le même que le premier. Dans la nuit du 25 au 26 février, il rend son âme à Dieu et, selon son vœu exprimé dans son testament, il est inhumé dans l'église des Récollets. Il meurt célibataire. Dans le relevé de ses effets personnels on fait mention d'un journal ou diaire des années 1676, 1677 et 1678 ainsi que de plusieurs autres papiers sans date ${ }^{76}$. Personne n'a malheureusement trouvé trace de ces documents. Le tout, a-t-on présumé, avait été expédié au Sieur de La Tourette en France. Vaudreuil, qui annonce à Pontchartrain la mort de Greysolon Dulhut, dit de lui: "c'était un très honnête homme". Charlevoix affirme également qu'il était "un des plus braves officiers que le roi eut en cette colonie" 7 .

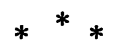

La postérité a-t-elle été juste envers cet homme et l'histoire lui a-t-elle donné la place qui lui revient? Henri Lorin, auteur d'une étude approfondie sur Frontenac, veut voir en Dulhut un "découvreur au même titre que La Salle". C'est encore à La Salle que le compare Louise Phelps Kellogg, dans son livre sur l'ouest américain, décernant même à Dulhut une réelle supériorité sur La Salle en bien des points ${ }^{78}$. Opinion partagée par Grace Lee Nute, autre auteur américain de renom ${ }^{79}$. Dulhut ne jouit peut-être pas du même prestige au Canada. Pourquoi?

Les voyages de Dulhut sont moins importants et surtout moins connus que ceux de La Salle. Il n'a d'ailleurs laissé aucun compte rendu tant soit peu considérable de ses explorations. Très absorbé,

76. William McLennan, "The Death of Duluth", Mémoires de la Société Royale section II (Toronto, 1903), 41s.

77. R.P. L. Le Jeune, Dictionnaire général du Canada (2 vol. Ottawa, 1931).

78. Louise Phelps Kellogg, French Regime in Wisconsin and the Northwest (Madison, 1925), 207.

79. Grace Lee Nute, Lake Superior (New York, 1924), 30. 
au surplus, par la traite des fourrures, - ce qui lui valut l'accusation de Duchesneau et les invectives de La Salle - son rôle de grand explorateur et de pacificateur indien a été relégué dans l'ombre. Dulhut n'eut pas, non plus, comme La Salle, des amis puissants à la Cour: un abbé Bernou, un Cardinal d'Estrées, une Madame de Maintenon ${ }^{80}$. Il ne semble pas avoir possédé, au moins au même degré, le coloris, l'élan, l'impétuosité héroïque de La Salle. Il l'égala pourtant en courage et en sang-froid et son empire sur les Indiens fut probablement plus profond et surtout plus durable. Ainsi il parvint à pacifier des tribus en guerre depuis des générations; il réussit à faire accepter, par les Sioux, l'allégeance française; il ajouta à l'empire français cette partie du Mississipi, si riche en fourrures et celle, encore plus riche peut-être, du nord et de l'ouest du lac Supérieur. Grâce à lui traitants et explorateurs purent pénétrer plus tard dans ces régions et y renouveler des alliances précieuses; celle des Sioux, par exemple, cimentée par Dulhut, se continua à travers les vicissitudes du régime français. Si Dulhut n'a pas été un fondateur d'empire ni un conquistador, il a été un précurseur, humain et juste, un héraut de la civilisation française, un fidèle et dévoué serviteur de son pays.

Antoine d'Eschambault, ptre de la Société historique de Saint-Boniface (Manitoba).

80. Grace Lee Nute, Caesars of the Wilderness (New York, 1943), 159. 